\title{
The relative role of ErbB1 -4 receptor tyrosine kinases in radiation signal transduction responses of human carcinoma cells
}

G Bowers, D Reardon, T Hewitt, P Dent, RB Mikkelsen, K Valerie, G Lammering, C Amir and RK Schmidt-Ullrich

Oncogene (2001) 20, $1388-1397$

The above paper was wrongly published as a 'Review' article. The correct section heading for this paper is 'Original Paper'. 